\title{
Risk of Recurrent Zoonotic Pandemic due to increasing Human-Animal Interface
}

\author{
Md. Azizul Haque ${ }^{1}$
}

In recent decades, we have noticed globally increasing human-animal interface and emergence of novel viral diseases such as severe acute respiratory syndrome (SARS), avian influenza A/H7N9, H5N1, Middle East respiratory syndrome (MERS); Nipah virus disease; Ebola hemorrhagic fever, Influenza A (H1N1) 2009 pandemic and the current COVID-19 pandemic. $^{1,2}$

Zoonotic origin of large-scale outbreak was documented in SARS in 2002. SARS started in the Guangdong province of China, and with time, it spread over 29 countries, affecting 8422 cases and causing 916 deaths. SARS-Cov was documented in Himalayan palm civets and Raccoon dogs. Rapid identification and segregation of the intermediate amplifying hosts from humans led to successful containment of this outbreak, and finally, the pandemic ended within seven months of its origin. ${ }^{3}$

The middle-east witnessed the outbreak of MERS in 2012. Saudi Arabia was the country of origin; however, gradually, it spread to Europe, Asia, Africa, and North America. As of July 2015, 1368 laboratory-confirmed cases of MERS, including at least 487 deaths (with a $35 \%$ case fatality rate), have been reported to $\mathrm{WHO} .{ }^{4}$

MERS CoV originated from bats, but camels acted as an intermediate transmission source to humans. ${ }^{5}$

Although most human cases of MERS-CoV infections have been attributed to human-to-human infections in health care settings, current scientific evidence suggests that dromedary camels are a major reservoir host for MERS-CoV and an animal source of MERS infection in humans. However, the exact role of dromedaries in the transmission of the virus and the same route(s) of transmission are unknown. Ebola virus disease is another deadly zoonotic disease occurring in several African countries for the last 40 years. The case fatality rate of Ebola ranges from $60-90 \%$, categorizing it as a category A priority pathogen. Ebola may be spread as a result of handling bushmeat (wild animals hunted for food) and contact with infected bats. The disease infects humans through close contact with infected animals, including chimpanzees, fruit bats, and forest antelope. After that, human-to-human transmission occurs. $^{6}$

The largest outbreak of Ebola in West Africa started from Guinea and then spread to Sierra Leone, Liberia. It took two years (2014-2016) to control the outbreak. $^{7}$

Another potentially deadly zoonotic disease affecting South-East Asia is the Nipah virus. Initially originated in Malaysia and Singapore in 1998, where Nipah virus spread from fruit bats to pigs and then from pigs to humans. The initial outbreak continued till June 1999 and was aborted by slaughtering millions of pigs. ${ }^{8}$

Nipah virus outbreaks have been recognized in Bangladesh since 2001. Fruit bats of Pteropus species have been identified as the natural reservoir of the Nipah virus. Unlike Malaysia and Singapore, the Nipah virus in Bangladesh is directly transmitted from fruit bats to humans without any intermediate host. The high case fatality rate and absence of efficacious treatment or vaccine make Nipah virus outbreaks a severe cause for concern in Bangladesh and India. ${ }^{9}$

${ }^{1}$ Associate Professor, Department of Medicine, Rajshahi Medical College, Bangladesh. 
In mid-April 2009, the Centers for Disease Control and Prevention and the World Health Organization had recognized a dramatic increase in the number of influenza cases. It was caused by a new strain of influenza type A H1N1 virus which is a reassortment of several strains of influenza viruses commonly infecting human, avian, and swine populations. This evolution was quite dependent on the swine population, which acted as the main reservoir for the reassortment event in the virus. ${ }^{10}$

In March 2010, more than 200 countries reported confirmed cases of Influenza A (H1N1) 09, and the global death count in that pandemic rose to $16,931 .{ }^{11}$

The ongoing pandemic caused by SARS-CoV-2 is another addition to the list of zoonotic diseases, putting humankind under an unprecedented level of danger. As of 27th January 2022, 366,918,707 confirmed cases of COVID-19, including 5,656,960 deaths, have been reported. ${ }^{12}$

Bats have been mentioned as their natural reservoir as it is known to harbor a variety of CoVs, including SARS-CoV- and MERS-CoV-like viruses. The coronavirus identified from Malayan Pangolins had a similarity of 91.02 percent to SARS-CoV-2, indicating the probability of Pangolins being the intermediate host of SARS-CoV-2. ${ }^{13}$

Rapid deforestation, shrinking of boundaries between human and animal, crisis for natural habitation, increasing demands for wildlife products are the causes of increasing human-animal contact. Large quantities of animal waste generated due to animal husbandry may also allow rapid selection, amplification, dissemination of zoonotic pathogens and facilitate zoonotic pathogen adaptation to the human body. Unfortunately, the global public health system is not adequately equipped to contain such epidemics due to inadequate understanding, poor preparedness, lack of interdisciplinary approach in surveillance and control strategy, and deficient political commitments.

The current covid-19 pandemic not only endangered human life but also massively affected the global economy, increasing the rich-poor divide and putting the healthcare delivery system of the marginalized countries on the brink of collapse. Humankind will also require a lot of time to recover from the covid infodemic. Moreover, the politicalizing covid pandemic will leave a long-lasting scar in the human psyche, resulting in the potential erosion of trust in scientific advances.

Albert Camus has written in his notebooks- "Real generosity towards the future lies in giving all to the present." Therefore, we must utilize the lessons learned from the ongoing pandemic to prevent upcoming zoonotic pandemics.

\section{References}

1. Cherry JD, Krogstad P. SARS: The first pandemic of the 21st century. Pediatr Res. 2004;56:1-5.

2. World Health Organization. (2003). Consensus document on the epidemiology of severe acute respiratory syndrome (SARS). https://apps.who.int/iris/handle/10665/70863

3. Guan Y, Zheng BJ, He YQ, Liu XL, Zhuang ZX, Cheung $\mathrm{CL}$, et al. Isolation and characterization of viruses related to the SARS coronavirus from animals in southern China. Science. 2003;302:276-8.

4. Chan JF, Li KS, To KK, Cheng VC, Chen H, Yuen KY. Is the discovery of the novel human betacoronavirus 2c $\mathrm{EMC} / 2012$ (HCV-EMC) the beginning of another SARSlike pandemic? J Infect. 2012;65:477-89

5. Han HJ, Yu H, Yu XJ. Evidence for zoonotic origins of Middle East respiratory syndrome coronavirus. J Gen Virol. 2016;97(2):274-280. doi:10.1099/jgv.0.000342

6. Reward S, Mirdha D. Transmission of ebola virus disease: an overview. Ann Glob Health. 2014 Nov-Dec;80(6):44451

7. Briand S, Bertherat E, Cox P, Formenty P, Kieny MP, Myhre JK, et al. The international Ebola emergency. N Engl J Med. 2014;371:1180-3

8. Centers for Disease Control and Prevention (CDC). Update: outbreak of Nipah virus -Malaysia and Singapore, 1999. MMWR Morb Mortal Wkly Rep. 1999;48:335-7.

9. International Centre for Diarrheal Diseases Research Bangladesh. Person-to-person transmission of Nipah infection in Bangladesh. Health Sci Bull. 2007;5:1-6.

10. Khanna, M., Kumar, B., Gupta, N. et al. Pandemic swine influenza virus (H1N1): A threatening evolution. Indian J Microbiol 2009; 49: 365-369

11. World Health Organization. Evolution of a pandemic $\mathrm{A}(\mathrm{H} 1 \mathrm{~N} 1) 2009$

12. Worldometer. Coronavirus. [accessed on January 28 , 2022]. Available from: https://www.worldometers.info/coronavirus/

13. Zhang $T, W u Q$, Zhang Z. Probable Pangolin origin of SARS-CoV-2 associated with the COVID-19 outbreak. Curr Biol. 2020;30:1351.e2. erratum in Curr Biol 2020:30;1578.

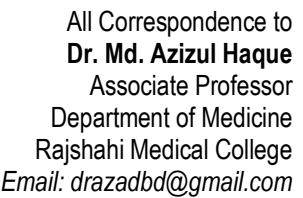

All Correspondence to

Dr. Md. Azizul Haque

Department of Medicine

Email: drazadbd@gmail.com 